\title{
La Alfabetización Informacional (ALFIN), usando Plataforma Virtual de Enseñanza y Aprendizaje en Educación Superior
}

\author{
Isabel C. Cordeiro de B. ${ }^{1}$ - María T. Mancilla B. ${ }^{2}$ - Grisneldy M. Reyes R. ${ }^{3}$ - Marisa A. Lara E. ${ }^{4}$ \\ Investigadoras / Universidad de Los Lagos, Chile \\ Recepción: 28-09-2015 / Aceptación: 10-11-2015
}

\section{Resumen}

Este artículo da cuenta del desarrollo del proyecto, "Plan de mejoramiento de los recursos de aprendizaje, usando nuevas tecnologías en los tres Campus de la Universidad de Los Lagos", en el marco del proyecto MECESUP ULA 1109, denominado Programa de mejoramiento de la calidad y equidad de la Educación Superior. Las acciones de este proyecto se focalizaron en la creación de competencias informacionales, las cuales - una vez finalizada la ejecución y evaluación del proyecto - fueron insertas en los módulos de programas de estudio de asignaturas transversales TIC que ofrece la institución. Las competencias han sido desagregadas en tres niveles de desarrollo, con el fin de facilitar su aprendizaje. Para cada nivel de las competencias se asocian los contenidos implicados en el desarrollo de las habilidades antes mencionadas, con sus correspondientes resultados de aprendizaje.

Durante un semestre académico, las competencias, niveles de logro, contenidos, habilidades y resultados de aprendizaje, se incorporaron en las asignaturas transversales TIC, utilizando la Plataforma de Enseñanza y Aprendizaje (PLATEA) de la institución, a modo de pilotaje. Por otra parte, se resalta el compromiso social de las bibliotecas universitarias para educar a través de ALFIN, colaborando en el desarrollo de este tipo de competencias. En este sentido, los bibliotecólogos, agregando a su trabajo tradicional de Procesos Técnicos, Referencia y Circulación, apoyan la labor educativa, aportando junto a los docentes a los procesos formativos de los estudiantes.

Se explicita que para promover el desarrollo de competencias informacionales en los estudiantes universitarios, es necesario el trabajo colaborativo entre bibliotecólogos y docentes. Además, se describe el proceso de implementación de ALFIN, los contenidos y algunos resultados.

Palabras clave: Alfabetización en Información, ALFIN, Bibliotecas Universitarias, Educación en Información, Plataformas Educativas, Competencias Transversales

\begin{abstract}
This article is about the development of the Project "Plan for Improvement of the learning resources, using new technologies in the three campuses of University of Los Lagos", as part of the Project MECESUO ULA 1109, called program for the improvement of quality and equity of Higher Education. The actions of this Project are focused on the creation of informal competencies, which once the implementation and evaluation of the Project were finished, they were embedded in the curriculum modules of the transversal ICT courses that are offered by the institution. The competencies have been broken down into three levels of development, in order to facilitate the learning. For each competency level, contents implied in the development of the competencies are associated in the development of the aforementioned competencies, and their corresponding learning results.
\end{abstract}

During one academic semester, the competencies, levels of achievement, contents, skills and learning results, are incorporated in the transversal ICT subjects using the Teaching and Learning Platform (TEALEA) of the institution, as a pilot. Moreover, the social commitment of the university libraries to teach through ALFIN is highlighted, assisting with the development of this type of competencies. In this way, librarians, adding to their traditional work of Technical Processes, References and Circulation, support the educational work with professors, providing the training process for the students.

It makes it clear that to promote the development of information skills in college students, it is necessary the collaborative work between librarians and teachers. In addition, the implementation process of information literacy, content and some results are described.

Key Words: Information Literacy, ALFIN, University Libraries, Education in Information, Education Platforms, Transversal Competencies

1. Bibliotecóloga, Magíster en Bibliotecología e Información, Universidad Playa Ancha, Valparaíso, Chile. Bibliotecóloga de la Universidad de Magallanes. Email: isabel.cordeiro@umag.cl

2. Bibliotecóloga Jefe de la Biblioteca Central “Pablo Neruda”, de la Universidad de los Lagos. Email: mtmancil@ulagos.cl

3. Académica, Magíster en Ciencias de la Educación, Mención Curriculum y Evaluación, Universidad de Los Lagos. Osorno, Chile. Email: greyes@ ulagos.cl

4. Académica, Magíster en TICs en Educación: Análisis y diseño de procesos, recursos y prácticas formativas, Universidad de Salamanca. Directora del Centro de Recursos de Enseñanza y Aprendizaje (CREA), de la Universidad de los Lagos, Campos Osorno, Chile. Email: mlara@ulagos.cl 


\section{Introducción}

La "Educación de Usuarios” es un servicio que tradicionalmente está presente en las bibliotecas universitarias, siendo uno de sus propósitos educar a través de las actividades ofrecidas por éstas unidades de información, para desarrollar autonomía y competencias en el uso de los recursos existentes. Para ejemplificar esta actividad de formación, cabe aquí mencionar la "inducción", donde usualmente se orienta sobre la ubicación de los textos, utilización del préstamo interbibliotecario, recomendación del uso de catálogos, entre otros temas.

Con la aparición de las TIC, el volumen actual de información, así como la difusión del conocimiento, se hacen cada vez mayor, favoreciendo la democratización del acceso a la información y la generación de conocimiento, demandando de nuevas habilidades en los usuarios para saber buscar, localizar y utilizar un gran número de fuentes de información, tornándose imperativo saber determinar las mejores fuentes y evaluar los contenidos.

La disponibilidad de variados recursos de información digital, como las bases de datos bibliográficos representan nuevos y complejos retos, ya que los usuarios deben tener las competencias para saber buscar y recuperar los documentos electrónicos que se encuentran almacenados de la forma más ágil y efectiva posible. En este sentido, resulta fundamental la labor formativa del Bibliotecólogo como un educador en información.
Hoy día, varias bibliotecas universitarias no sólo imparten el tradicional servicio de "Educación de Usuarios", sino que además, cuentan con programas educativos en el ámbito de la Alfabetización Informacional, donde se educa en torno a la información. Este programa de alfabetización constituye un reto actual para las bibliotecas universitarias, las que deben promover el desarrollo de competencias informacionales, donde el usuario es demandado ante la necesidad de saber usar estrategias eficaces de búsquedas, de utilización de distintas fuentes para recuperar información, tener conocimiento de cómo se aplica los criterios para evaluar, ser reflexivo y crítico con la información y de cómo se registra la información y sus fuentes.

Según el argumento de la actual Sociedad de la Información, el desarrollo de la Alfabetización Informacional estaría colaborando con competencias necesarias para la formación del pensamiento crítico, aprender a aprender (a lo largo de toda la vida) y aportar a la nueva cultura.

El porqué de estas necesidades se refleja en una diversidad de argumentos, destacándose lo mencionado por Basulto Ruíz (2009, p.15), donde afirma que según la Comisión Europea (2001), «el aprendizaje permanente es considerado un factor clave frente a la incertidumbre económica, el aumento de las desigualdades y la exclusión social»

Por otra parte, este mismo autor (2008, p.6) al referirse a la Alfabetización Informacional, afirma: «está encaminada hacia el "aprender a aprender", hacía la adquisición de habilidades 
o competencias para que un individuo pueda actuar de manera autónoma en un contexto creciente de información».

En el ámbito de la enseñanza superior, tratándose de la necesidad de la Alfabetización Informacional, González Flores (2012, p. 68) afirma que:

Las universidades tienen el deber de formar profesionales de excelencia capaces de adquirir, compartir y transmitir conocimiento durante toda su vida, que no estén preparados únicamente para un tipo de trabajo; que puedan adaptarse a las nuevas circunstancias que impone una sociedad cambiante y que evoluciona con gran rapidez, que sean capaces de captar nuevas ideas y conocimientos, así como de evaluar de forma crítica, que se cuestionen las cosas y no puedan vivir en la incertidumbre.

En este contexto, la Universidad de Los Lagos, a través del Proyecto Mejoramiento de la Calidad y Equidad en la Educación Superior, Universidad de Los Lagos (MECESUP ULA 1109) ofrece dicha oportunidad para los estudiantes de esta casa de estudios. A partir de ello se han desarrollado competencias informacionales, tomando en cuenta el compromiso social y el nuevo rol del bibliotecólogo; agregando a su trabajo tradicional de Procesos Técnicos, Referencia y Circulación, la labor educativa; aportando junto a los docentes en los procesos formativos, e impulsando el desarrollo de competencias en información, donde se ha explicitado, en esta Universidad, como un saber transversal institucional.
Tal como afirma la bibliotecóloga Catalán Chávez (2013, p.5):

Es importante que los estudiantes aprendan a conocer sus necesidades de información y por ende, que aprendan a acceder a ella, pero también es importante que los docentes y los bibliotecólogos se unan, para que los estudiantes aprendan a usarla correctamente.

Considerando la afirmación anterior, y que las bibliotecas universitarias deben estar insertas en los procesos educativos de las instituciones superiores, podemos confirmar que estas unidades deben desarrollar en los usuarios las competencias en información.

Por lo tanto, esta nueva dimensión de biblioteca universitaria va más allá de su función tradicional, siendo una guía y orientador de aprendizajes relacionados con las búsquedas eficientes de información, la elaboración de bibliografías y uso de bases de datos; siendo éstas oportunidades una instancia para trabajar directamente con estudiantes universitarios y docentes. A partir de ello se generan saberes integrados y desempeños en el tratamiento de la información, que implican el desarrollo de capacidades y habilidades esenciales en el proceso de enseñanza aprendizaje, en el cual están insertos. Pero, ¿cómo se llega a concretar estos propósitos?, ¿cómo nace dicha labor formativa?, ¿cómo podrán ser agentes efectivos para propiciar aprendizajes?, ¿cuáles son las acciones, tiempos y recursos específicos para llevar a cabo el programa de Alfabetización Informacional en la Universidad de los Lagos? 
Antes de responder a estas preguntas, se debe definir el término Alfabetización Informacional, también conocida por la sigla ALFIN. Cabe destacar que, en la literatura existente hay una gran diversidad de opiniones al respecto, por lo que a continuación mencionamos una definición aclaratoria, la expresada por Basulto Ruíz (2009, p.3), donde comenta los sucedido en la reunión de expertos sobre la Alfabetización Informacional (ALFIN), congregados en Praga (2003). Ellos consensuaron que:

la alfabetización informacional comprende el conocimiento y necesidades de los individuos y la habilidad para identificar, localizar, evaluar, organizar y crear, utilizar y comunicar información eficazmente para enfrentar aspectos o problemas, en un prerrequisito para participar eficazmente en la Sociedad de la Información y es parte de los derechos básicos de la humanidad para un aprendizaje de por vida.

\section{Contexto}

Actualmente estamos insertos en la Sociedad de la Información, donde se hace más necesario que nunca su uso inteligente para poder aprender, trabajar y tomar decisiones. Quienes forman parte de esta sociedad, se caracterizan por el uso significativo de información y por el uso de las Nuevas Tecnologías de la Información.

La cantidad excesiva de información disponible es una de las características de esta so- ciedad. Considerando este actual escenario donde es imperativo la obtención de competencias para el acceso y su uso adecuado, varios autores se refieren a este tema con preocupación, es el caso de Leiva (2003, p.223), donde afirma:

Actualmente, el problema no es conseguir información, sino qué hacer con el gran volumen disponible. Sin lugar a dudas este fenómeno sugiere la adquisición de habilidades de estudiantes y profesores para buscar información, reconocer qué información se requiere e identificar cuál es necesaria y al mismo tiempo conocer herramientas para acceder y aprovechar las facilidades de conectividad que cada día son mayores en los centros educativos y en la sociedad en general.

Por lo tanto, para participar de esta Cultura Informacional es necesario estar alfabetizado informacionalmente; es decir, poseer las habilidades necesarias para utilizar los datos de manera autónoma durante toda la vida, valiéndose de la tecnología disponible, conscientes de la importancia de saber cómo se aprende; del pensamiento crítico y de la aplicación de lo que se sabe, según el contexto social en que se vive, pues el saber leer y escribir no es lo único fundamental.

Matus Sepúlveda y Fibla R. (2004, p.23), nos hace referencia de cuales habilidades $y$ competencias se requiere. Las autoras afirman: «Una persona alfabetizada en información debe:

5. Hacia una sociedad informacionalmente alfabetizada. Declaración de Praga. Traducida del inglés por Gloria Ponjuan. 


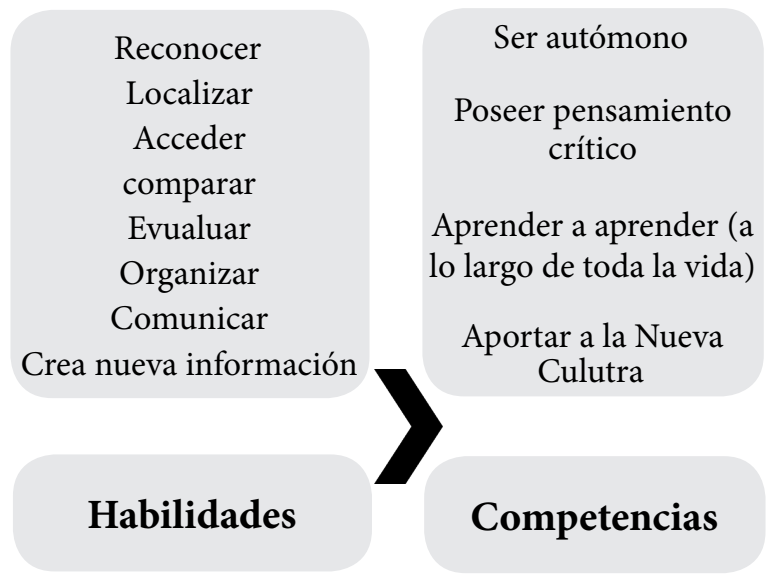

Figura 1. comparación de habilidades y competenicias.

En el ambiente de la enseñanza superior, el estudiante universitario necesita desarrollar dichas habilidades informacionales, de tal forma que le permitan obtener competencias útiles en su vida académica, profesional y personal.»

La Alfabetización Informacional refuerza éste proceso de formación en cada estudiante, desarrollando las habilidades y competencias que le son requeridas.

\section{¿Para qué les sirve ALFIN a los Universitarios?}

- Para fomentar la generación del conocimiento.

- Para desarrollar habilidades y competencias informacionales a lo largo de toda la vida.

- Para ayudarles en la búsqueda y recuperación de información para sus necesidades académicas.

- Para valorizar la información.
¿Cómo nace y se implementa el Programa de Alfabetización Informacional (ALFIN) en la Universidad de los Lagos?

Dado estos requerimientos, la Alfabetización Informacional se puso en marcha desde julio 2013, se integró dentro de las Competencias Transversales en TIC en la Universidad de Los Lagos a las carreras de Pedagogía en Educación Media, mención Historia y Geografía; Pedagogía en Educación Media, mención Inglés y Traducción; Educación Diferencial, un módulo que fue montado sobre la Plataforma de Enseñanza y Aprendizaje PlatEA, la cual permite un aprendizaje teórico-práctico de dichas competencias.

\section{Metodología}

Para ello se diseñaron - por parte de un equipo multidisciplinario - los ambientes y materiales instruccionales; objetos de aprendizaje, tutoriales, guías, evaluaciones y autoevaluaciones en línea, con un acompañamiento web por el experto disciplinar. También se desarrollaron talleres presenciales en la Biblioteca Central de la Universidad, respondiendo con ello a las necesidades de Formación Integral del Estudiante.

Para la implementación de este programa, inicialmente se nombró un Coordinador y se formó un equipo de trabajo interdisciplinario, donde se realizaron diferentes reuniones de trabajo entre el personal de Biblioteca Central y algunas unidades pertenecientes a la Dirección de Docencia de esta casa de estudios, como la Unidad de Formación Integral 
(UFIN), Unidad de Desarrollo Docente y Curricular (UDEDOC) y el Centro de Recursos de Enseñanza y Aprendizaje (CREA); así como también con docentes que impartían los módulos que tributan al desarrollo de las competencias transversales TIC en la institución.

Los objetivos del Módulo ALFIN son:

\section{Objetivo General:}

Desarrollar habilidades y competencias informacionales que les permita a los estudiantes ser autónomo en la búsqueda, recuperación y uso de la información para satisfacer necesidades académicas y profesionales.

\section{Objetivos Específicos:}

- Resaltar la importancia de la información en el mundo actual.

- Desarrollar habilidades de información necesarias para el proceso de aprendizaje.
- Plantear la necesidad de poseer pensamiento crítico frente a la informacion.

- Dar a conocer los recursos disponibles en Biblioteca y en Internet.

- Crear una cultura de uso eficiente de la información.

El área curricular de la Unidad de Desarrollo Docente y Curricular (UDEDOC), junto a la Biblioteca Central, determinó los niveles de competencias ALFIN, que a continuación se detallan:

A. Competencia 1: Gestiona la información impresa y digitalizada de materias científicas y de intereses personales, accediendo a ésta eficientemente y utilizándola de forma creativa, crítica y responsable.

\begin{tabular}{|c|c|c|}
\hline Nivel & Contenidos & Resultados de aprendizaje \\
\hline $\begin{array}{l}\text { I. Conocimiento de los recursos } \\
\text { disponibles en el Sistema de Biblio- } \\
\text { tecas de la universidad. }\end{array}$ & $\begin{array}{l}\text { - Características de la Sociedad de } \\
\text { la Información. } \\
\text { - Importancia de las Competen- } \\
\text { cias Informacionales. } \\
\text { - Servicios y recursos disponibles } \\
\text { en el Sistema de Biblioteca (Web } \\
\text { biblioteca, catálogo en línea, prés- } \\
\text { tamos inter bibliotecarios). }\end{array}$ & $\begin{array}{l}\text { - Explica por qué es importante la } \\
\text { alfabetización informacional en } \\
\text { la Sociedad del Conocimiento. } \\
\text { - Localiza la información a través de } \\
\text { los recursos disponibles en la Web. }\end{array}$ \\
\hline $\begin{array}{l}\text { II. Aplica y analiza las bases de da- } \\
\text { tos bibliográficas con información } \\
\text { científica a nivel local y de otros } \\
\text { centros de información. }\end{array}$ & $\begin{array}{l}\text { - Utilización de las diversas bases de } \\
\text { datos bibliográficas. } \\
\text { - Uso de acertadas estrategias de } \\
\text { búsquedas: uso de conceptos y pa- } \\
\text { labras claves para acceder a docu- } \\
\text { mentos relevantes. }\end{array}$ & $\begin{array}{l}\text { - Emplea estrategias de búsqueda } \\
\text { para recuperar información } \\
\text { - Selecciona la información pertinente. }\end{array}$ \\
\hline
\end{tabular}




\begin{tabular}{|l|c|c|}
\hline \multicolumn{1}{|c|}{ Nivel } & \multicolumn{1}{|c|}{ Contenidos } & Resultados de aprendizaje \\
\hline & $\begin{array}{c}\text { Búsquedas avanzadas: conectores } \\
\text { booleanos, meta-buscadores, fra- } \\
\text { ses exactas. }\end{array}$ & $\begin{array}{c}\text { Organiza la información de acuer- } \\
\text { do a la necesidad de aprendizaje. }\end{array}$ \\
$\begin{array}{l}\text { III. Resuelve problemas relaciona- } \\
\text { dos con la revisión y el análisis de } \\
\text { distintas fuentes de información. }\end{array}$ & $\begin{aligned} \text { - Aplicación de las normas para reali- } \\
\text { zar bibliografías, según su titulación. }\end{aligned}$ & $\begin{array}{l}\text { Utiza las normas internacionales } \\
\text { de bibliografía para citar las fuentes } \\
\text { impresas y electrónicas. }\end{array}$ \\
& $\begin{array}{l}\text { Coherencia entre la necesidad de } \\
\text { aprendizaje requerida y la infor- } \\
\text { mación recopilada y analizada. }\end{array}$ & $\begin{array}{l}\text { Comunica la nueva información } \\
\text { de manera pertinente a las situa- } \\
\text { ciones de aprendizaje. }\end{array}$ \\
\hline
\end{tabular}

Figura 2. Competencia y sus niveles Uno

B. Competencia 2: Emprende iniciativas relacionadas con el proceso de alfabetización informacional con el fin de obtener aprendizajes significativos que satisfagan sus necesidades académicas, personales y profesionales, a partir de un trabajo autónomo y eficiente.

\begin{tabular}{|c|c|c|}
\hline Nivel & Contenidos & Resultados de aprendizaje \\
\hline $\begin{array}{l}\text { I. Comprende la importancia de } \\
\text { poseer iniciativa para la búsque- } \\
\text { da de información que ayude en } \\
\text { la toma de decisiones y soluciones } \\
\text { de problemas. }\end{array}$ & $\begin{array}{l}\text { - Identifica fuentes primarias y } \\
\text { secundarias. } \\
\text { - Distinción entre información } \\
\text { relevante. }\end{array}$ & $\begin{array}{l}\text { - Con autonomía, identifica las dis- } \\
\text { tintas fuentes de información, } \\
\text { esenciales para resolver necesida- } \\
\text { des informacionales. }\end{array}$ \\
\hline $\begin{array}{l}\text { II. Aplica la autonomía para uso } \\
\text { de información, examinándola con } \\
\text { pensamiento crítico. }\end{array}$ & $\begin{array}{l}\text { - Criterios para evaluar la informa- } \\
\text { ción encontrada (autoría, fuente, } \\
\text { actualización, etc.). } \\
\text { - Actitud crítica y reflexiva ante la } \\
\text { información. }\end{array}$ & $\begin{array}{l}\text { - Juzga con criterio para evaluar la } \\
\text { información, utilizando pensa- } \\
\text { miento crítico. }\end{array}$ \\
\hline $\begin{array}{l}\text { III. Resuelve con eficiencia proble- } \\
\text { mas relacionados con los recursos } \\
\text { de información disponibles }\end{array}$ & $\begin{array}{l}\text { - Uso ético y responsable de la infor- } \\
\text { mación: distinción y análisis entre } \\
\text { propiedad intelectual y plagio. } \\
\text { - Utilización de fuentes primarias } \\
\text { y secundarias para propósitos } \\
\text { específicos } \\
\text { - Uso de estructura para la elabora- } \\
\text { ción de un informe de investigación. }\end{array}$ & $\begin{array}{l}\text { - Actúa y argumenta frente a di- } \\
\text { lemas éticos relacionados con } \\
\text { la información, reflejando res- } \\
\text { ponsabilidad. } \\
\text { - Utiliza competencias informacio- } \\
\text { nales de organización, comuni- } \\
\text { cación y revisión de información } \\
\text { encontrada o creada, con eficiencia } \\
\text { y autonomía. }\end{array}$ \\
\hline
\end{tabular}

Figura 3. Competencia y sus niveles Dos.

El grupo de trabajo, que tiene la tarea de implementar este programa informacional, establece que las competencias deben ser insertas en los módulos de programas de estudio de asignaturas transversales TIC, que ofrece la institución. Se determinó que las competencias fue- 
ran desagregadas en niveles de desarrollo, con el fin de facilitar el aprendizaje. Para cada nivel de las competencias, se asocia los contenidos implicados en el desarrollo de las habilidades antes mencionadas, con sus correspondientes resultados de aprendizaje.

Posteriormente, Biblioteca Central conjuntamente con el Centro de Recursos de Enseñanza Aprendizaje (CREA), se encargaron de elaborar y aplicar un Pre Test, que fue titulado como "Cuestionario de Verificación de Competencias ALFIN", para diagnosticar las competencias y habilidades tecnológicas e informacionales de los estudiantes. Al término del módulo, se volvió a aplicar el mismo documento para saber el nivel de competencia individual de cada estudiante.

Para la validación del instrumento se solicitó asesoría a bibliotecólogos externos, especialistas en Alfabetización Informacional, enviando la solicitud de evaluación vía correo electrónico con el link del cuestionario por evaluar.

Biblioteca buscó ejemplos de tutoriales y guías en Internet, sobre la Alfabetización Informacional para seleccionar temas del módulo
Alfabetización Informacional (ALFIN). Terminada dicha tarea, gracias a la valiosa ayuda del Centro de Recursos de Enseñanza Aprendizaje (CREA), se elaboró el diseño gráfico del módulo, dejando virtualmente disponible en la Plataforma de Enseñanza Aprendizaje, los contenidos relacionados con el primer nivel de la primera y segunda competencias para que fueran aplicados como piloto. Enseguida, se definieron las carreras con las que se trabajarían las competencias ALFIN durante el piloto.

La difusión del programa se desarrolló mediante página web institucional y de Biblioteca; a través de trípticos para distribuir con la Comunidad Universitaria, y entre los usuarios de Biblioteca. Finalmente, se comenzó a impartir el módulo ALFIN durante un semestre académico; las competencias, niveles de logro, contenidos, habilidades y resultados de aprendizaje, han sido incorporadas en las asignaturas transversales TIC a modo de pilotaje.

A partir de entonces, hasta el día de hoy, se continúa con el propósito de ofrecer el módulo completo según semestre, niveles y competencias (Ver figura 4).

\begin{tabular}{|c|c|c|}
\hline Semestre & Nivel & Competencia \\
\hline Primer & 1 & 1 y 2 \\
\hline Segundo & 2 & 1 y 2 \\
\hline Tercero & 3 & 1 y 2 \\
\hline
\end{tabular}

Figura 4. Niveles de Competencias 


\section{Proceso de Implementación}

A continuación, detallamos el resumen del proceso de implementación para la Alfabetización Informacional en la Universidad de los Lagos:

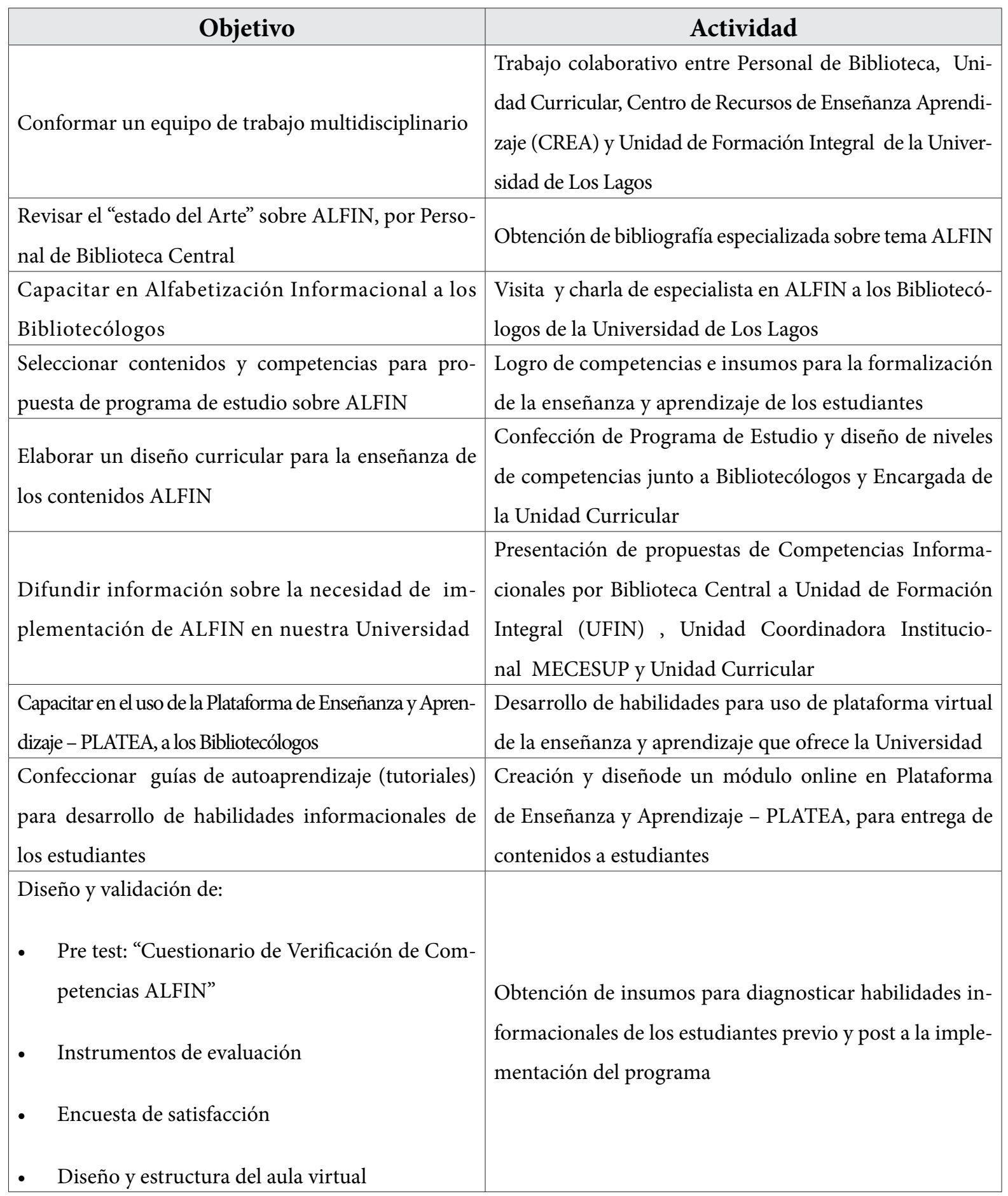




\begin{tabular}{|l|l|}
\hline \multicolumn{1}{|c|}{ Objetivo } & \multicolumn{1}{c|}{ Actividad } \\
\hline $\begin{array}{l}\text { Generar espacios de discusión, evaluación y bús- } \\
\text { queda de consensos en torno a la implementación de } \\
\text { ALFIN en nuestra institución }\end{array}$ & $\begin{array}{l}\text { Reunión con Docentes para impartir ALFIN como mó- } \\
\text { dulos transversales TIC en programas de estudios }\end{array}$ \\
\hline $\begin{array}{l}\text { Generar instancias de formación para el desarrollo } \\
\text { de competencias ALFIN }\end{array}$ & $\begin{array}{l}\text { Disponibilidad de contenidos sobre ALFIN en platafor- } \\
\text { ma virtual de enseñanza de nuestra Universidad (PLA- } \\
\text { TEA), desde Centro de Recursos para el Aprendizaje } \\
\text { (CREA) }\end{array}$ \\
\hline $\begin{array}{l}\text { Difundir información sobre ALFIN en medios } \\
\text { de comunicación }\end{array}$ & $\begin{array}{l}\text { Elaboración de contenidos para Página Web de Bibliote- } \\
\text { ca, entre otros insumos }\end{array}$ \\
\hline Diseñar material de difusión sobre tema ALFIN & $\begin{array}{l}\text { Obtención de producto para sociabilización del tema } \\
\text { con Comunidad Universitaria }\end{array}$ \\
\hline
\end{tabular}

Figura 5. Competencia y sus niveles Uno

\section{Respecto del acceso a los contenidos del módulo ALFIN a través de la Plataforma de Enseñanza y Aprendizaje (PlatEA)}

A través de la plataforma virtual (figura 6) se hace posible el acceso a los contenidos del Programa ALFIN en la Universidad de los Lagos, logrando así generar una instancia de aprendizaje para el desarrollo de competencias y habilidades informacionales.

Esta plataforma virtual de aprendizaje posibilita que el estudiante pueda acceder y trabajar en los contenidos del módulo en horario diurno o nocturno, según la disponibilidad de tiempo.

Para una mayor aclaración, mencionamos que

Es una plataforma e-learning, que permite a docentes (profesores, tutores, ayudantes...) la creación y administración de cursos, quedando disponibles para los estudiantes. El rol del profesor es crear el contenido del curso, proponer actividades, etc., y el estudiante es la persona que recibirá el conocimiento y realizará las actividades de aprendizaje propuestas. Además, la plataforma proporciona el aprendizaje autónomo de los estudiantes y permite reforzar la habilidad de la comunicación efectiva con los participantes.

Es un espacio interactivo, donde las actividades formativas son guiadas por un bibliotecólogo, tutor virtual experto en contenidos, contando además con un tutor online que se encarga del seguimiento y monitoreo del proceso formativo dentro de la plataforma del curso virtual.

El diseño del aula de este curso virtual fue realizado por un profesional del Centro de Recursos de Enseñanza Aprendizaje (CREA), donde previamente se presentó al equipo de trabajo la propuesta de diseño instruccional,

6. Disponible en: http://platea.ulagos.cl/ 
interfaz del aula virtual, la ruta de navegación y acceso de los alumnos al curso, disposición y estructura de los módulos, formas de evaluación, tipos de evaluación y propuesta de validación del curso en general.

Respecto de la propuesta del diseño del aula virtual, se contemplaron los siguientes ítems:

Introducción: Presentación de los aspectos formales del curso cómo, nombre del módulo, espacio de bienvenida, programa módulo o asignatura, formas de evaluación, tipos de evaluación, reglamento del aula virtual, recomendaciones del trabajo, espacio de consultas, orientación para el estudiante tanto del aula como la ruta de aprendizaje.

Diseño del formato del curso: Ubicación de bloques, interfaz, colores, imágenes, fondos, logos etc.

Diseño diagrama del curso: Semanal, espacio de bienvenida para el estudiante, organización de los contenidos, iconografía de orientación.

Espacio de documentación: Contempla archivos, cómo guías paso a paso o tutoriales para acceder y realizar acciones dentro del aula; cómo crear su perfil, subir su foto, subir un archivo etc.

Testeo Plataforma: Se revisaron los elementos dispuestos en el Curso.

Se realizó una Inducción de la Plataforma PlatEA a los estudiantes que participaron del Programa ALFIN; los responsables de la Inducción fueron docentes del Centro de Recursos CREA y bibliotecólogas. Se presentó el acceso, manejo y uso de las herramientas tecnológicas y las formas de evaluación dispuestas en el aula virtual, las bibliotecólogas realizaron la presentación de las temáticas de ALFIN.

Los estudiantes demostraron estar receptivos a la modalidad de implementación del módulo; se planificaron y acordaron en conjunto con los estudiantes las tutorías tanto presenciales como virtuales. La finalidad de implementar tutorías presenciales es que permita a los estudiantes contar con espacios para aclarar dudas sobre los contenidos, u otros temas relacionados con el uso de dicha plataforma. Además, para la tutoría se creó y utilizó la red social WhatsApp para la comunicación entre estudiantes, docentes y bibliotecólogos. Cabe aquí mencionar que para el acceso, cada estudiante y profesor tiene su propia clave.

\section{Plataforma implementación Módulo ALFIN}

Link de acceso a la Plataforma PlatEA: http://platea.ulagos.cl/

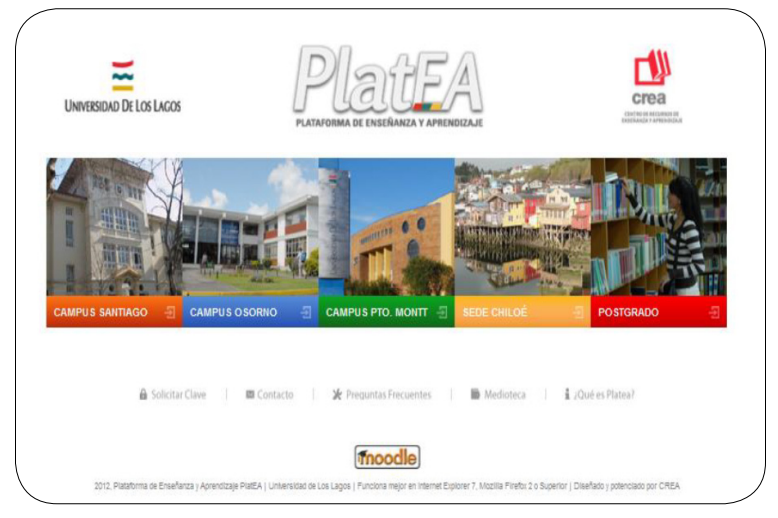

Figura 6. Pantalla inicial Plataforma PlatEA.

Los estudiantes y docentes cuentan con accesos según el Campus o Sede al cual pertenecen. Aquí se muestran los Campus de pregrado Osorno, Puerto Montt y Santiago de Chile, Sede Chiloé e Instituto Tecnológico Regional Osorno y Puerto Montt. 


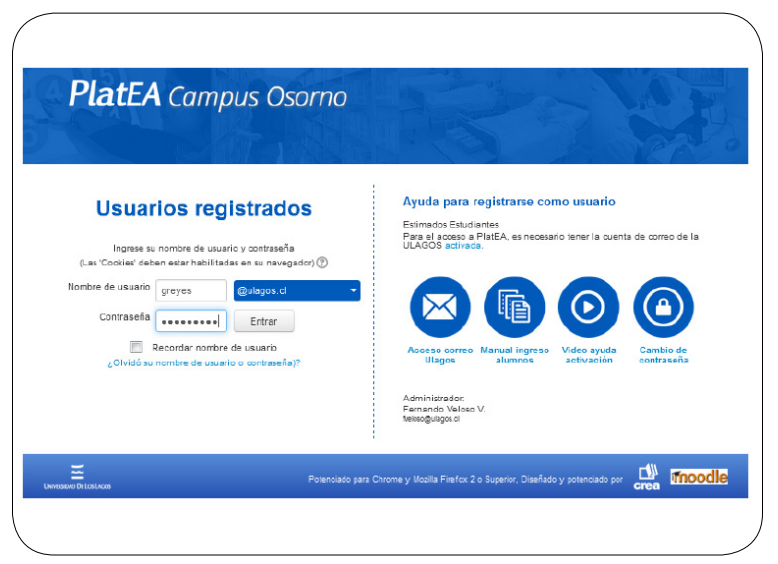

Figura 7. Muestra de acceso de estudiantes y académicos a la Plataforma Virtual PlatEA. Se requiere ingresar nombre de usuario y contraseña.

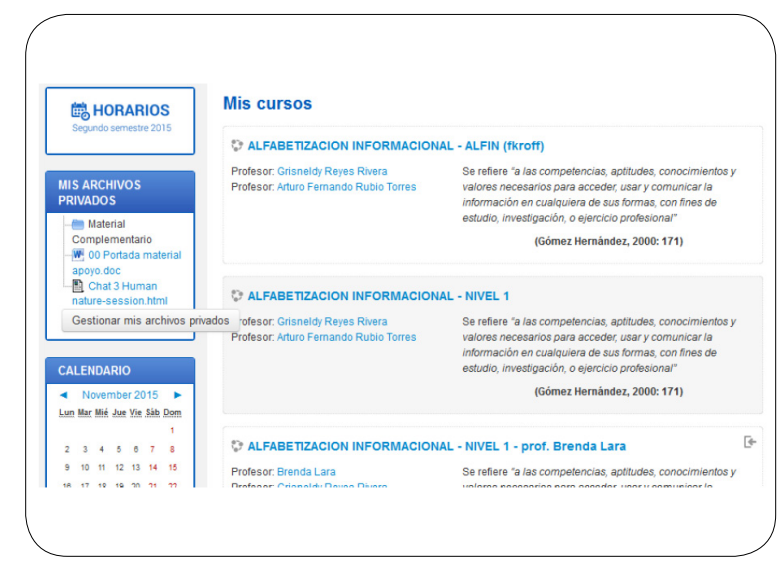

Figura 8. Imagen de acceso de estudiantes y académicos a los cursos disponibles en la Plataforma PlatEA.

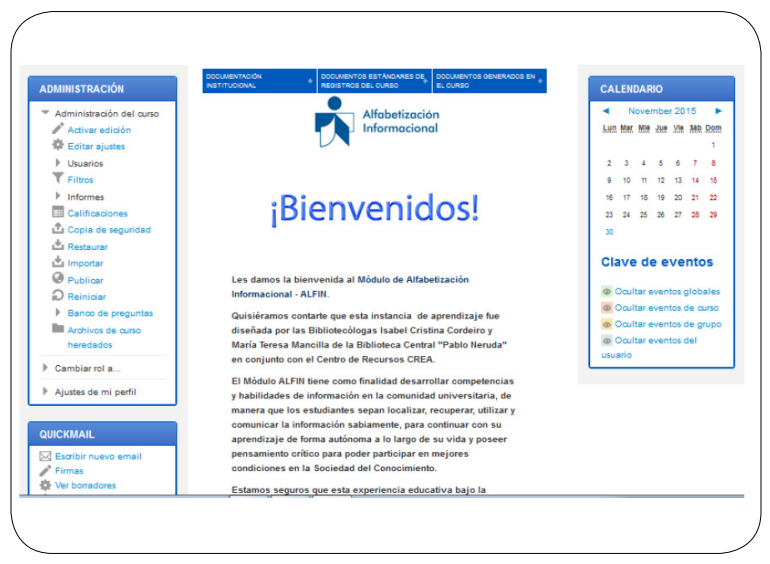

Figura 9. Muestra de interfaz del módulo ALFIN. Espacio de Bienvenida al curso.

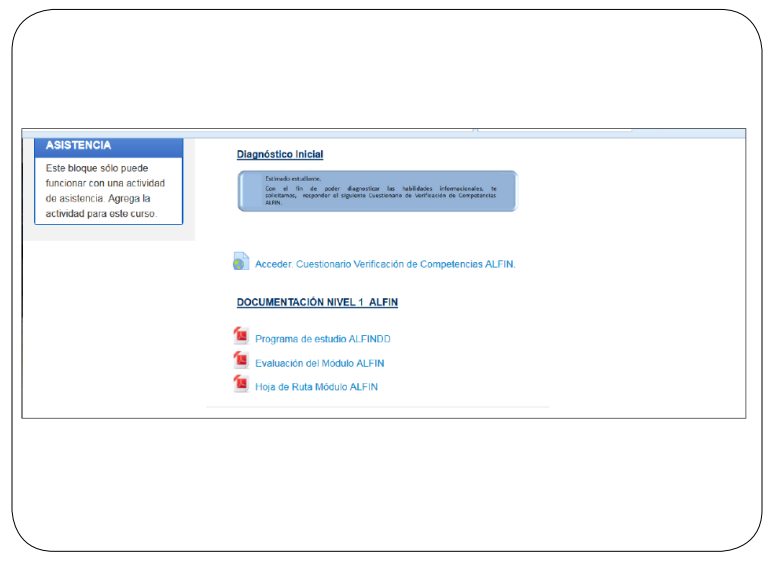

Figura 10. Imagen del espacio de acceso a los contenidos del módulo ALFIN del aula del curso. Los estudiantes pueden acceder al cuestionario de Verificación de Competencias ALFIN, que consistía en una encuesta en línea; también a la documentación curricular del módulo, contemplaba el programa de asignatura del módulo, los tipos de evaluación y la ruta de aprendizaje. 


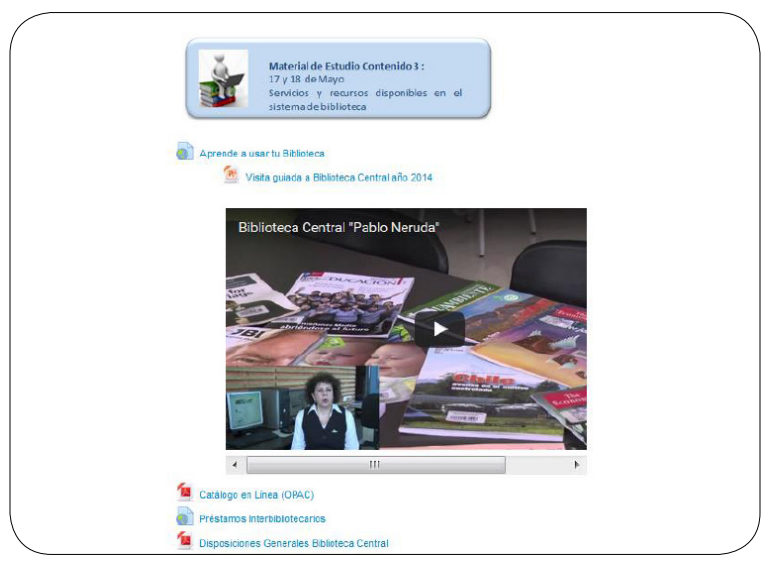

Figura 11. Acceso a espacio de contenidos del curso. La documentación que se dispuso en el aula virtual se encuentra en diferentes formatos para facilitar a los estudiantes el autoaprendizaje, y una mayor comprensión de los contenidos tratados según el nivel de la competencia declarada y los resultados de aprendizaje que han sido declarados. Para la sección contenidos del curso se dispusieron tutoriales, contenidos en formtao pdf; y también diseño de contenidos en el programa presentaciones PowerPoint, en el cual se insertaron links para revisar y videos tutoriales.

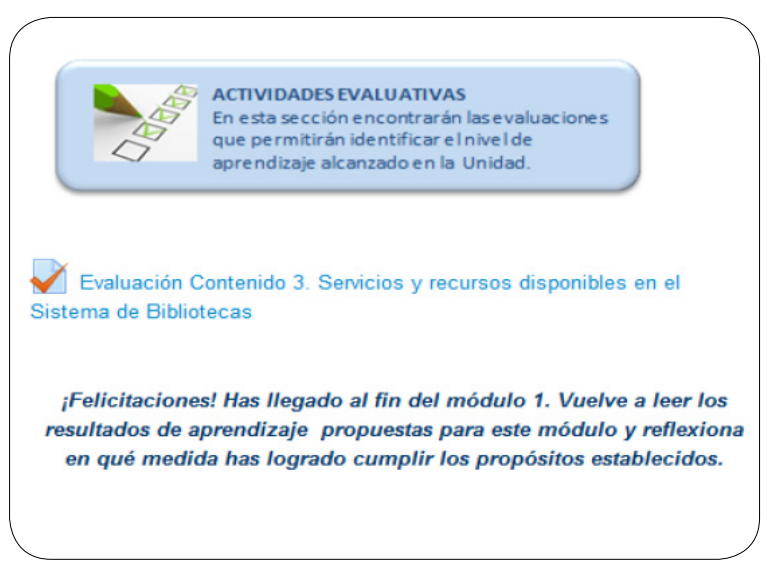

Figura 12. Espacios de tutoría en línea. Una vez que los estudiantes revisaban los contenidos del curso de manera autónoma, paralelamente se realizaban las tutorías tanto presenciales como virtuales, en horarios y fechas previamente acordadas con los estudiantes.

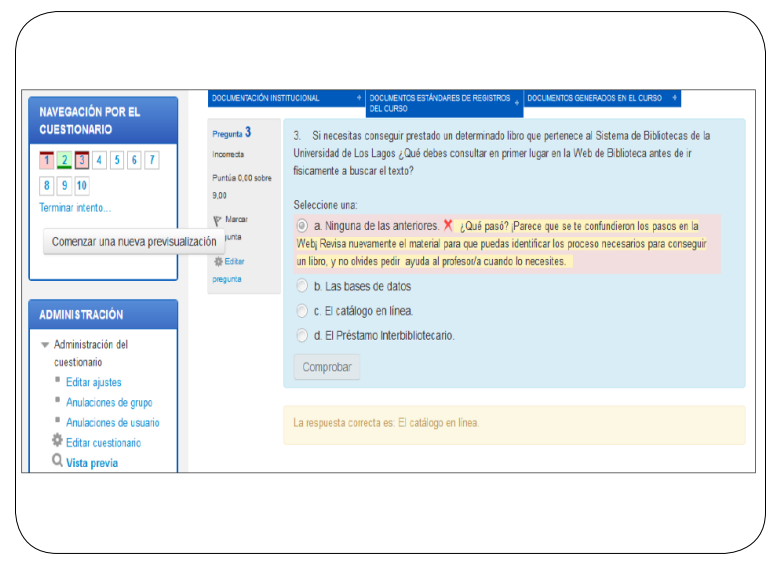

Figura 13. Se contemplaba dentro de los instrumentos de evaluación la retroalimentación inmediata, permitiendo al estudiante ir revisando su avance y progreso, así como identificar los errores.

\section{Validación del Aula Virtual}

Los contenidos fueron aportados por las bibliotecólogas expertas; por tanto, no fue necesario someter los contenidos a validación de jueces externos.

Se validó el diseño instruccional del curso virtual, tanto la interfaz de presentación de los contenidos, como la estructura formal del curso y la ruta de aprendizaje, validados por el Área Curricular/UDEDOC de la dirección de Docencia.

Además, algunos docentes expertos en el área Informática educativa, contaron con acceso al curso virtual para poder hacer sugerencias, observaciones y correcciones que permitieron mejorar tanto la interfaz del aula, navegación de los elementos dispuestos, disposición y estructura de los contenidos. 


\section{Resultados}

Participaron de la aplicación del módulo de Alfabetización Informacional tres carreras de pregrado del área educativa: Educación Diferencial, Pedagogía en Educación Media mención Historia y Geografía; y Pedagogía en Educación Media mención Inglés y Traducción. La muestra estuvo compuesta por un total de 95 estudiantes (ver figura 14), que tuvieron acceso a los contenidos del módulo dispuesto en PlatEA.

\begin{tabular}{|c|c|c|c|}
\hline Carreras & Semestre & $\begin{array}{l}\text { Nombre } \\
\text { asignatura }\end{array}$ & $\begin{array}{l}\text { Cantidad } \\
\text { estudiantes }\end{array}$ \\
\hline Educación diferencial & I semestre & Computación básica & 16 \\
\hline $\begin{array}{l}\text { Pedagogía en ed. Media, } \\
\text { mención historia y geo- } \\
\text { grafía }\end{array}$ & I semestre & Computación básica & 7 \\
\hline $\begin{array}{l}\text { Pedagogía en ed. Media, } \\
\text { mención historia y geo- } \\
\text { grafía }\end{array}$ & V semestre & $\begin{array}{l}\text { Desarrollo de software } \\
\text { educativo }\end{array}$ & 13 \\
\hline $\begin{array}{l}\text { Pedagogía en ed. Media, } \\
\text { mención inglés y traduc- } \\
\text { ción }\end{array}$ & I semestre & Computación básica & 17 \\
\hline $\begin{array}{l}\text { Pedagogía en ed. Media, } \\
\text { mención inglés y traduc- } \\
\text { ción }\end{array}$ & III semestre & $\begin{array}{l}\text { Informática educativa y } \\
\text { creación de multimedios }\end{array}$ & 27 \\
\hline $\begin{array}{l}\text { Pedagogía en ed. Media, } \\
\text { mención inglés y traduc- } \\
\text { ción }\end{array}$ & V semestre & $\begin{array}{l}\text { Desarrollo de software } \\
\text { educativo }\end{array}$ & 15 \\
\hline \multicolumn{3}{|r|}{ Total } & 95 \\
\hline
\end{tabular}

Figura 14. Carreras de pregrado participantes del Módulo ALFIN (Nivel 1 Competencia 1 y $2)^{7}$, como piloto, en el mes de julio a diciembre de 2013.

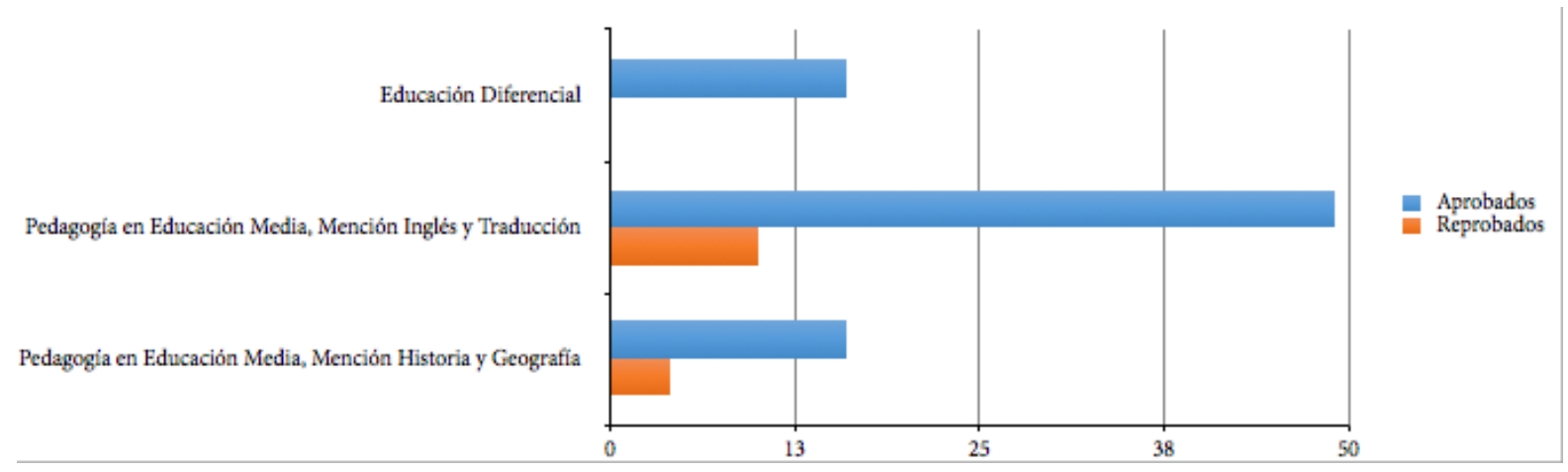

Figura 15. Resultados de evaluaciones de estudiantes pregrado participantes en Módulo Alfabetización Informacional, Nivel 1 Competencia 1 y 2.

7. Ver tabla 1: Competencia y sus Niveles 1 y tabla 2: Competencia y sus niveles 2. 
a. Evaluación del Uso de PlatEA, en la ejecución del curso virtual del Programa ALFIN

Con el propósito de realizar una evaluación de este recurso, se aplicó a los estudiantes una encuesta de satisfacción de manera anónima, en línea y voluntaria, con el objeto de orientar las mejoras factibles a este programa educativo.

La selección de sujetos informantes para realizar esta evaluación se realizó considerando el siguiente criterio de selección ya preestablecido:

Estudiantes que participaron del Módulo ALFIN (Nivel 1 Competencia 1 y 2) ) $^{8}$ como piloto, desde el mes de julio hasta diciembre 2013.

La encuesta se llevó a cabo en Diciembre del año 2013, ya que en este mes se finalizaba el término del semestre. La bibliotecóloga tutora, junto a una profesional docente, informaron previamente en cada curso, que estaba participando del Programa ALFIN, sobre la aplicación de dicha encuesta, y solicitaron la colaboración de los estudiantes para responderla. Posteriormente, dicha encuesta fue puesta en línea a través de la Plataforma virtual PlatEA, estando a la disposición de ellos por dos semanas. Se solicitó verbalmente, con correos electrónicos y WhatsApp, que respondan con seriedad, ya que dicho estudio era de interés común, pues los datos recolectados beneficiarían en las acciones para la mejoría e innovación del programa ALFIN.

La encuesta abarcó sólo unas pocas preguntas y fueron respondidas por medio de alter- nativas, así como también, se dio la posibilidad de que expresaran sus opiniones, si así lo deseaban. Del universo de 95 estudiantes, sólo 34 de ellos respondieron la encuesta de satisfacción que corresponde al 36\% de la población encuestada.

\section{Análisis de resultados}

El análisis de los resultados contemplados en la encuesta se realizó con base en las siguientes categorías:

\begin{tabular}{|l|}
\hline Categorías \\
\hline Contenidos del módulo \\
\hline Aspectos temporales \\
\hline Las evaluaciones \\
\hline Funcionamiento de la plataforma \\
\hline Interfaz del curso \\
\hline Las tutorías \\
\hline
\end{tabular}

Figura 16. Categorías del programa.

Considerando los siguientes criterios:

\begin{tabular}{|l|}
\hline Categorías \\
\hline Muy de acuerdo \\
\hline De acuerdo \\
\hline Ni de acuerdo Ni en desacuerdo \\
\hline En descuerdo \\
\hline Muy en desacuerdo \\
\hline No contemplado \\
\hline
\end{tabular}

Figura 17. Criterios de evaluación del programa.

El resultado del estudio se plantea de acuerdo a las categorías y utilizando los criterios de evaluación ya mencionados.

El análisis de los datos tiene el siguiente orden: se presenta las preguntas que tienen relación

8. Ver tabla 5: Carreras de pregrado participantes del Módulo ALFIN (Nivel 1 Competencia 1 y 2, como piloto, en el mes de julio a diciembre 2013. 
con las categorías, se hace un análisis cuantitativo con la presentación de los gráficos donde de reflejan las opiniones de los estudiantes respecto a la encuesta aplicada y, posteriormente, se analizan los resultados obtenidos.

A dos categorías abordadas (los contenidos del módulo y funcionamiento de la Plataforma), se agregaron algunos comentarios expresados por los estudiantes al responder a una pregunta abierta de la encuesta. Estas respuestas nos permiten realizar un análisis cualitativo, permitiendo así la descripción de las percepciones de los involucrados acerca de los temas tratados.

\section{Categoría: Contenidos del módulo}

Al analizar la encuesta que se aplicó a los estudiantes, mediante la pregunta: ¿Los contenidos disponibles en el curso permiten comprender la importancia de adquirir competencias informacionales?, el resultado se puede expresar en el siguiente gráfico:

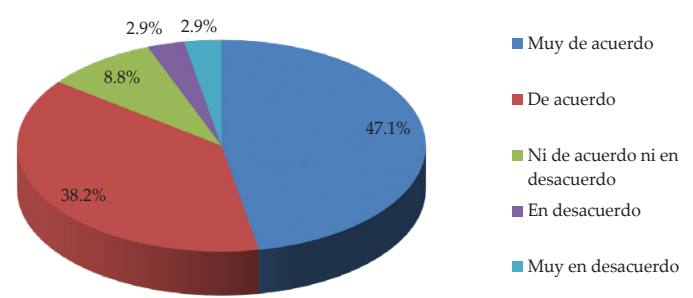

Figura 18. Contenidos del módulo.

Como se observa en el gráfico, un mayor porcentaje de estudiantes consideró que los contenidos trabajados en el módulo de Alfabetización Informacional (ALFIN) permiten comprender la importancia de adquirir competencias informacionales.
Este resultado valida la importancia de incluir la ALFIN en los módulos de los programas de estudio de asignaturas transversales TIC, que ofrece la Universidad de Los Lagos, contribuyendo así que sus estudiantes accedan a los contenidos, desarrollando las competencias necesarias ya propuestas, ya que los contenidos tratados en dicho módulo, pasan a ser parte de la formación académica de ellos. También sirven como un "chapuzón” para que comiencen a valorar los temas relacionados con la información y el conocimiento, tan importantes en el mundo actual.

A continuación se mencionan algunos párrafos, en donde los estudiantes plantean su parecer sobre los contenidos del módulo.

«Fue bueno el propósito del curso, ya que en mi caso personal pude comprender cosas que antes no tomé en cuenta o desconocía»

«Creo que los contenidos fueron entregados de manera fácil de entender, de forma bien didáctica, para familiarizarnos con las competencias informacionales»

«Todo muy bueno permite adquirir más conocimiento sobre un tema para mí, casi desconocido»

Se demuestra que, efectivamente, los contenidos abordados en el Programa ALFIN, satisficieron las expectativas de los estudiantes.

\section{Categoría: Aspectos temporales}

Al analizar la siguiente pregunta: ${ }_{¿}$ El tiempo disponible para la revisión de los contenidos del curso fue suficiente para su análisis 
y estudio?, el resultado se encuentra en el siguiente gráfico:
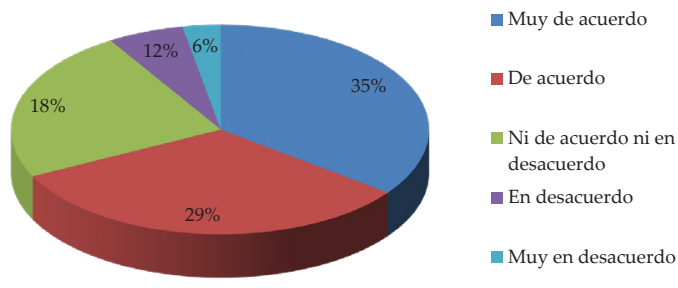

Figura 19. Aspectos temporales.

En este gráfico se ve claramente que el mayor porcentaje de estudiantes, considera que el tiempo disponible para revisar los contenidos del curso es suficiente para la comprensión de los temas tratados.

Este resultado valida la importancia de incluir el programa ALFIN en la Plataforma de Enseñanza y Aprendizaje de la Universidad (PlatEA), dado que este recurso está al alcance de sus estudiantes en cualquier momento, las 24 horas del día; disponible desde cualquier punto donde haya conexión a Internet. Esta flexibilidad para el acceso a la información, pasa a ser un factor positivo para el estudiante, ya que éste pasa a ser autónomo en la obtención de su aprendizaje.

\section{Categoría: Las evaluaciones}

Con la pregunta, ¿Las evaluaciones te permitieron medir el nivel de conocimiento adquirido?, el resultado está en el gráfico que se encuentra a continuación:

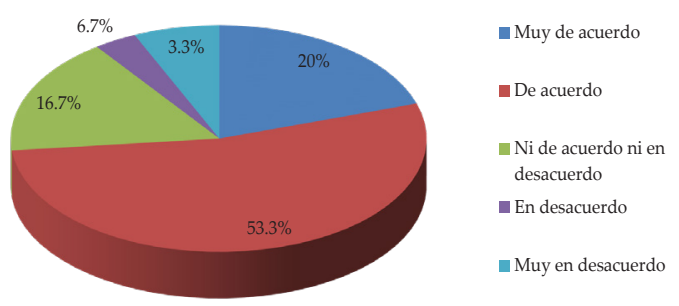

Figura 20. Las evaluaciones.

Según lo que se aprecia en el gráfico, respecto a las evaluaciones en línea, los estudiantes consideraron que los instrumentos y las preguntas que fueron plateadas, les permitieron medir el nivel de conocimiento y el aprendizaje que han logrado alcanzar en cada una de las unidades.

Este resultado es positivo, debido a que evidenció que los estudiantes quedaron satisfechos con los conocimientos obtenidos a través de las evaluaciones realizadas.

\section{Categoría: Funcionamiento de la Plataforma}

En la pregunta: ¿La plataforma es amigable y fácil de usar?, el resultado se encuentra en el gráfico a seguir:
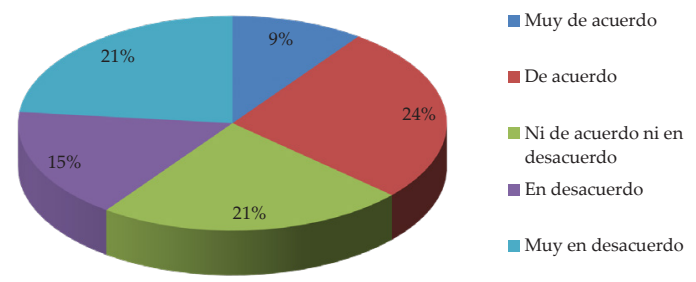

Figura 21. Funcionamiento de la Plataforma. 
El gráfico nos demuestra que PlatEA presenta algunos inconvenientes, lo ideal es que posea sistemas interactivos más amigables, que al ser utilizadahalla mejor "navegabilidad" (por ejemplo, que nopresente lentitud) para una mayor satisfacción del uso de este recurso.

A continuación, se mencionan algunos párrafos de la encuesta, en donde algunos estudiantes se refierenal Funcionamiento de la Plataforma, estasapreciaciones nos permiten realizar un análisis cualitativo.

\section{Los estudiantes dicen:}

- "La verdad es que la PLATAFORMA efectivamente es fácil de utilizar, sin embargo el acceso es deficiente, muchas veces simplemente resulta imposible conectarse."

- “Todo bien, lo único que dificultó responder la encuesta fue platea y sus continuos problemas.”

- "La plataforma tiene muchas fallas, colapsa rápido."

Este resultado fue positivo, debido a que evidencia que los estudiantes quedaron satisfechos con los conocimientos obtenidos a través de las evaluaciones realizadas. En la opinión de los estudiantes, se requiere mejorar la conectividad en PlatEA, permitiendo una mayor rapidez en su uso.

\section{Categoría: Interfaz del curso}

En la pregunta: ¿Los recursos disponibles en el curso (tales como imágenes, textos, videos, infografías, etc.) facilitaron la comprensión de los contenidos? Se obtuvo el siguiente gráfico:

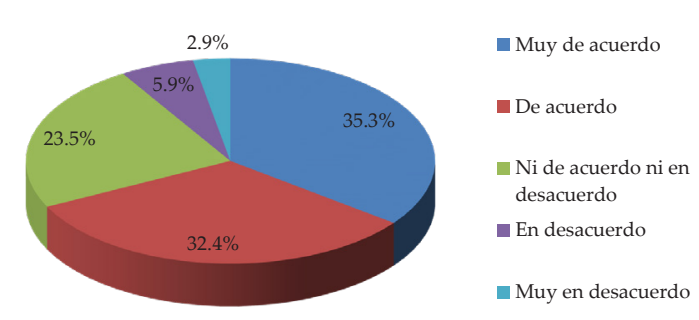

Figura 22. Interfaz del curso.

Considerando este gráfico, se puede señalar que la mayoría de los elementos que se colocaron a disposición del aula virtual de aprendizaje, tales como: imágenes, textos, videos e infografías facilitaron la comprensión y el autoaprendizaje del módulo. Esto demuestra que los estudiantes están conformes con los recursos de información disponibles en el módulo ALFIN.

El resultado refleja que contar con recursos apropiados para el acceso a los contenidos es un requisito valioso cuando se está utilizando la plataforma; además sirve para ayudar en la comprensión de los contenidos.

\section{Categoría: Las tutorías}

Al analizar la pregunta: ¿La comunicación recibida por parte de las/los tutores/as motivó la participación en el curso?, se obtuvo el siguiente gráfico:

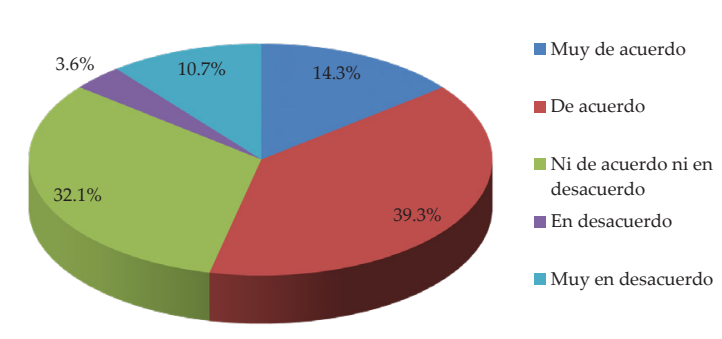

Figura 23. Las tutorías. 
Se constató que la comunicación recibida por parte los tutores motivó la participación en el curso de la mayoría de estudiantes, reflejándose la imperativa necesidad de que los tutores estén en constante contacto con los estudiantes, a través de todos los medios de comunicación pertinentes. Este contacto ayuda en profundizar los conocimientos, resolver dudas y a motivar la realización de las actividades programadas en el módulo.

$\mathrm{Al}$ analizar otra la pregunta relacionada con las tutorías:

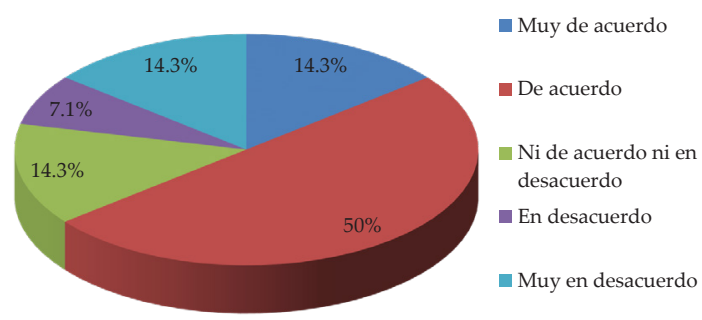

Figura 24. Las tutorías.

El gráfico refleja que la comunicación por parte de los tutores fue realizada frecuentemente durante el curso. Esta acción refuerza que se logran más beneficios respecto a las competencias, cuando el contacto con los tutores es regular.

\section{Discusión}

La Alfabetización Informacional dejó de ser un tema lejano de entender y se convierte en contenido necesario del aula. Estar capacitado solamente para buscar y recuperar información no es el único requisito para ser considerado experto en el uso de la misma. Lo que los estudiantes deben desarrollar es la consciencia acerca del valor de la información y su impor- tancia para la obtención del conocimiento; por lo tanto, los bibliotecólogos y docentes deben concienciar a los estudiantes respecto a esta imperativa necesidad.

La incorporación de la Alfabetización Informacional en la Universidad de Los Lagos pasa a ser una evolución de la formación del usuario de Biblioteca. Ello consiste en ir más allá de la enseñanza del uso de los recursos bibliográficos, para educar entorno a la información, promoviendo la toma de conciencia en los estudiantes - a objeto de adquirir habilidades y competencias informacionales-, de manera que sean autónomos y críticos en la búsqueda, recuperación y uso de la información.

La experiencia de implementación del Módulo ALFIN abre la puerta para implementar un sistema de este tipo, que sea soportado en la plataforma de enseñanza aprendizaje. Es necesario disponer de un sistema de navegación expedito a nivel institucional; es decir, instalar servidores con la capacidad suficiente para mantener conectados en forma simultánea a los estudiantes que hagan uso de los recursos que ahí se hospedan. Toda iniciativa tipo ALFIN requiere - para ser exitosa - continuidad; es decir, debe ser anclada en la estructura administrativa de la Institución, ya sea como Plan estratégico, Modelo Educativo Institucional, etc.

Esto es alentador, debido a que algunos estudiantes y profesores están trabajando con los contenidos relacionados a los temas más importantes para la sociedad actual; prevaleciendo la participación de los estudiantes en el proceso, generando discusión e inter- 
cambiando opiniones virtuales disponibles en la Plataforma de Enseñanza y Aprendizaje (PlatEA). También, a través de los talleres presenciales en Biblioteca Central, se generan los espacios para el desarrollo de habilidades y competencias informacionales necesarias en la actual «Sociedad de la Información».

Cualquier iniciativa de este tipo debe ser evaluada constantemente por parte de bibliotecólogos, académicos y estudiantes. Por otra parte, también es necesaria la constante actualización de los recursos disponibles y de los métodos de evaluación aplicados; además de mayor participación de académicos y bibliotecólogos en el grupo de trabajo de alfabetización informacional, para poder propiciar innovaciones y acciones formativas, potenciando así los logros de los objetivos ya propuestos. Además, es ideal que exista vinculación con el medio, en donde se puedan dar a conocer e impartir actividades relacionadas con el programa ALFIN a otros establecimientos educacionales de la región, dado que las bibliotecas necesitan adaptar sus servicios tradicionales al nuevo modelo social.

Insertar a los bibliotecólogos en el proceso de enseñanza-aprendizaje de la Universidad, les concibe asumir el compromiso social con la comunidad universitaria; sobre todo con los estudiantes. Esta acción parece ser un adecuado camino para la alfabetización informacional, tan necesaria en la actualidad.

Un trabajo colaborativo como este es necesario para establecer lazos entre bibliotecólogos y docentes de la Universidad. Estos últimos se han convertido en los aliados de la Biblioteca para el desarrollo de competencias, para el acceso, localización y uso adecuado de la información en los estudiantes; a la vez, los bibliotecólogos se convierten en colaboradores en los procesos de enseñanza-aprendizaje de sus estudiantes.

Se ha comprobado que, para educar sobre el tema Alfabetización Informacional, es necesario contar con ambientes educativos facilitadores de los procesos de aprendizajes, usando - además del ambiente físico de una biblioteca - otras opciones de recursos o instancias disponibles para educar entorno a la información, tales como las plataformas virtuales de enseñanza-aprendizaje.

Pero no sólo utilizar soportes tecnológicos es suficiente para el logro de las habilidades informacionales, también es importantísimo contar con el compromiso y el entusiasmo de los Bibliotecólogos, para impulsar servicios que contribuyan a la concientización respecto a la valoración de la información; y también el entusiasmo de los académicos, para inculcar, alertar e incentivar a sus estudiantes a optar por un aprendizaje autónomo en el ámbito informacional, en donde la búsqueda, acceso, uso y evaluación de la información sean acciones cotidianas para desarrollar el pensamiento crítico, la construcción de nuevos conocimientos y el logro de sus aprendizajes. Como resultado de esto, los alumnos lograrán un actuar inteligente en sus tareas académicas y/o en sus actividades diarias como ciudadanos en la Sociedad, impulsando así la educación permanente. 
Este estudio reveló que los objetivos de la implementación del programa ALFIN en la Universidad de los Lagos se cumplieron satisfactoriamente; y que la forma de implementación con la ayuda de la plataforma educacional PlatEA fue exitosa. Por otro lado, la planificación del Módulo ALFIN es adecuada a los fines de desarrollo de las competencias deseadas, pero aún existen acciones a mejorar, sobre todo en la lentitud de conexión on-line y el compromiso de los bibliotecarios y docentes para llevar a cabo esta formación tan necesaria en los estudiantes. Esto con la finalidad de perpetuar este proyecto como parte del currículum de todas las carreras.

Finalmente, se recomienda que otras institucionales educativas universitarias se sumen en esta tarea tan noble y necesaria, creando e implementado en sus casas de estudios programas educativos de ALFIN; incluso que sus bibliotecólogos y docentes realicen actividades conjuntas en otros establecimientos educacionales de enseñanza media y básica, para difundir la importancia de la información. De esta forma se formarán más y más ciudadanos capaces de apreciar la información y el conocimiento; insumos importantísimos para el desarrollo y el bienestar de la sociedad actual.

\section{Glosario}

- ALFIN: Alfabetización Informacional

- MECESUP: Mejoramiento de la Calidad y Equidad de la Educación Superior

- PlatEA: Plataforma de Enseñanza y Aprendizaje

- TIC: Tecnología de la Información y Comunicación

- UFIN: Unidad de Formación Integral

- ULA: Universidad de Los Lagos (Chile)

- UDEDOC: Unidad de Desarrollo Docente y Curricular 


\section{Referencias}

Basulto, E. (2008). Estrategia de alfabetización informacional para los estudiantes de la carrera de Ingeniería Agrícola que cursan el Plan de Estudios “D”. (Tesis). Universidad Agraria de La Habana, La Habana, Cuba. Recuperado de: http://eprints.rclis.org/12986/1/TM_EmiliaBasulto.pdf

Basulto, E. (2009). La alfabetización informacional. Revista Digital Sociedad de la Información. (16), jul. 1-32. Disponible en: http://www.sociedadelainformacion.com/16/alfabetizacion.pdf

Benito, F. (2006) “¿Qué es alfabetización informacional?”. Pinakes. Recuperado de: http://pinakes.educarex.es/numero3/descargas/colaboraciones2.pdf

Catalán, Y. (2013). Información científica, base de datos y alfabetización informacional (ALFIN), para todos. Recuperado de: http://eprints.rclis.org/20403/1/Art\%C3\%ADculo\%20en\%20Prensa\%20Y.Catal\%C3\%A1n.pdf

Gómez, J. (coord.) (2000). Estrategias y modelos para enseñar a usar la información: guía para docentes, bibliotecarios y archiveros. Murcia, K.R.

González, I. (2012). Necesidad de la Alfabetización Informacional en la educación superior [en Línea]. Revista de Comunicación Vivat Academia. Universidad de la Habana, Cuba. 15 (121), 65-76. Recuperado de: dialnet.unirioja.es/descarga/articulo/5098314.pdf

Leiva, D., Gaete, A. y Saurina, E. (2003). Habilidades para buscar, evaluar y usar información en estudiantes universitarios: una contribución a la "alfabetización informacional" de estudiantes de Educación. Revista Pensamiento Educativo, 32 (1), 221-249. Recuperado de: http://pensamientoeducativo.uc.cl/files/journals/2/articles/255/public/255-596-1-PB.pdf

Matus, G. y Fibla R., P. (2004). Alfabetización en información: una revolución en las Universidades. Recuperado de: http://www.cabid.cl/doctos/usuarios/u-playa-ancha.pdf

Matus, G. (2004). ¡Exijo ser infoalfabetizado! Sepiensa. Recuperado de: http://www.sepiensa.cl/ edicion/index.php?option=content $\&$ task $=$ view\&id=379\&Itemid $=43$

Matus, G. (2005). La influencia de un programa de intervención de formación de Usuarios autónomos en los procesos de enseñanza aprendizaje del alumnado de la Universidad de Playa Ancha, Valparaíso, Chile. Ciencias de la Información, 36(3). Recuperado de: http://cinfo.idict.cu/ index.php/cinfo/article/view/121/120

Reusch, A. (s.f.). La Alfabetización en Información: una necesidad desde la educación primaria. Valparaíso, Chile, Universidad de Playa Ancha. Recuperado de: http://iibi.unam.mx/publicaciones/19/tendencias_alfabetizacion_informativa-La\%20Alfabetizacion\%20en\%20Informacionanamaria-reush.html

Uribe-Tirado, A. (2011). Competência em informação: entrevista. Revista Brasileira de Biblioteconomia e Documentação,7(2), 194-203. Recuperado de: http://rbbd.febab.org.br/rbbd/article/ view/199/200 